\title{
8. ASSOCIATED UPPER-AIR EFFECTS
}

\author{
By C. W. Allen
}

The geophysical effects of solar flares give rise to one of the main applications of solar astronomy to every-day life. The effects themselves take place in the ionospheric levels and have been studied intensively by the methods of upper atmosphere research. However, there is some hesitation in interpreting results at the present time since our conclusions may soon need to be modified in order to take high-level rocket observations into consideration.

The flare effects(x), often called sudden ionospheric disturbances (S.I.D. or P.I.D.B.), might be listed (with abbreviations) as follows:

\begin{tabular}{|c|c|c|}
\hline S.W.F. & & Short-wave fade; observed on radio circuits. \\
\hline F.O. & A & Fade out; observed on ionospheric recorders. \\
\hline S.F.A. & & Sudden field strength anomaly; recorded on radio circuits. \\
\hline S.P.A. & A & Sudden phase anomaly. $16 \mathrm{kc} .(2)$ \\
\hline S.E.A. & A & Sudden enhancement of atmospherics. $27 \mathrm{kc} .(3,4)$ \\
\hline S.C.A. & A & Sudden cosmic noise anomaly. $18 \mathrm{Mc} / \mathrm{sec} .(5)$ \\
\hline C.C. & & Crochet curve.(6) \\
\hline I.A.M. & & Ionospheric absorption measurements. \\
\hline
\end{tabular}

There is a one-to-one correlation between flares and their effects and hence any clear S.I.D. observation can be taken as an indication of flare occurrence. The S.I.D.'s marked A can be measured quantitatively and thus give an opportunity of measuring flare intensities on a permanent and comparative basis. Observations of flares by means of S.I.D.'s could be carried out continuously without regard to weather by about three stations well separated in longitude. This type of observation has not beer developed and organized with the continuity it deserves and hence we do not have the systematic S.I.D. observing coverage that is desirable for solving certain solar-terrestrial problems.

Although there is a very close association between S.I.D.'s and flares, they are not completely simultaneous. The measured time differences (order of some minutes) have been used to determine the relaxation time for the level of the S.I.D. concerned $(x, 7,8$,$) .$ In order to interpret the time differences as ionospheric effects one must assume that the timing is the same for both the $\mathrm{H} \alpha$ emission and the ionizing radiation. If the ionization is due entirely to $\mathrm{L} \alpha$, this is probably a fair assumption but it is still difficult to devise a flare measurement (say flare area, $\mathrm{H} \alpha$ line width, or $\mathrm{H} \alpha$ central intensity) which can adequately represent the total emission.

In order to identify the wave-length of the ionizing radiation we may appeal to the relation between absorption coefficient of atmospheric gases and wave-length. For an atmosphere of known density the height of the ionized layer depends almost entirely on the absorption coefficient. Most of the S.I.D. effects occur below the E region and probably at a height of about $70 \mathrm{~km}$. (9). Using such a height in conjunction with a height against wave-length diagram (e.g. Goldberg (rof his Fig. 7), we find that the possible wave-lengths are in the neighbourhood of $5 \AA, L \alpha$, or rgoo $\AA$. We can discard $1900 \AA$ since it would not produce ionization and the choice falls between $\mathrm{L} \alpha$ and X-rays.

There can be very little doubt that $L \alpha$ plays a large part in S.I.D. ionization. Laboratory absorption measurements for $L \alpha(\mathrm{rr})$ agree with ionospheric measurements(x2) and lead to an ionization layer at about the right height. Also the increase in L $\alpha$ emission during a flare is reasonable. To see this we note that the $L \alpha$ flux as observed from rockets $(\mathrm{I} 2)$ is $0 \cdot \mathrm{I}$ erg $\mathrm{cm} .^{-2} \mathrm{sec}^{-1}$ (at earth). On the other hand, the radiation from a flare of extreme intensity can just produce disk brightening and might be presumed to have about $\mathrm{I} \%$ of the solar surface emission. Taking $1 \mathrm{IO}^{-3}$ of the Sun's disk as a representative great flare area the flux becomes ro erg $\mathrm{cm} .^{-2} \mathrm{sec} .^{-1}$ at earth; i.e. an increase factor of Ioo. A fairly similar result is obtained if we consider $\mathrm{H} \alpha$ emission intensity. Considering a class 2 flare in this case with $H \alpha$ equivalent breadth of $3 \AA$ and area $10^{-3}$ of the disk, and allowing a factor of Io for the greater energy and emission probability of $L \propto$ as 
compared with $\mathrm{H} \alpha$, we find an emission of $2 \mathrm{erg} \mathrm{cm} .^{-2} \mathrm{sec}^{-1}$. This gives a factor 20 increase for a class 2 flare. One could expect an increase of 100 or 20 to be readily detectable as a S.I.D. The only difficulty in the $\mathrm{L} \alpha$ explanation is that $\mathrm{L} \alpha$ has a singlevalued absorption coefficient and would produce a sharply bounded ionization layer. An $L \alpha$ increase of 20 or 100 times would cause a lowering of the layer of only 7 or $9 \mathrm{~km}$. (13) as compared with maximum observed falls of $15 \mathrm{~km}$. (2). This could readily be explained if $L \alpha$ were mixed with other effective radiations.

The part that may be played by X-rays in the formation of S.I.D.'s has been considered by Friedman and Chubb(13). A contribution of X-ray emission between $I$ and Io $\AA$ could complicate the $D$-region ionization as suggested by observations (9). Furthermore, X-rays of wave-length 6-8 $\AA$ have been observed in active regions (13) and it would then not be unexpected to find X-ray component emitted from flares.

\section{REFERENCES}

(I) M. A. Ellison, Pub. Roy. Obs. Edinburgh, 1, 53, 1950.

(2) R. N. Bracewell and T. W. Straker, M.N. ro9, 28, I949.

(3) M. A. Ellison, J. Atm. Terr. Phys. 4, 226, I953.

(4) M. Waldmeier, $Z . A P .26,205,1949$.

(5) C. A. Shain and A. P. Mitra, J. Atm. Terr. Phys. 5, 3r6, r954.

(6) D. H. McIntosh, J. Atm. Terr. Phys. I, 3I5, I95I.

(7) A. P. Mitra and R. E. Jones, J. Geoph. Res. 59, 39r, I954.

(8) A. P. Mitra and R. E. Jones, J. Atm. Terr. Phys. 4, I4I, I953.

(9) R. N. Bracewell and W. C. Bain, J. Atm. Terr. Phys. 2, 216, 1952.

(10) L. Goldberg, The Earth as a Planet, ed. Kuiper, p. 434, I954.

(I I) R. W. Ditchburn et al., Rocket Exploration of the Upper Atmosphere, ed. Boyd and Seaton, p. 327, 1954 .

(12) E. T. Byram et al., Phys. Rev. 9r, I278, 9953.

(13) H. Friedman and T. A. Chubb, The Physics of the Ionosphere, Phys. Soc. Conference, p. 58,1955 .

\section{DISCUSSION}

M. J. SeAton: The very biggest upper atmospheric storms (magnetic, ionospheric and auroral) are always associated with particularly large sunspot groups, but are they always preceded by flare phenomena?

C. W. AllEN: I would like to see a better organization for observing these relations. It is not known with certainty that flares do not precede the greatest disturbances. Some regular recording of S.I.D.'s is required to prove whether all storms are preceded by flares.

L. GOLDBERG: What is the wave-length of the X-rays penetrating to 70 or $80 \mathrm{~km}$.?

C. W. AlLEN : $2 \cdot 8 \AA$.

L. GolDBERG: The X-ray observations from rockets indicate greater wave-lengths.

C. W. Allen: They are 6-8 $\AA$, but no rocket observation has been made during a flare.

M. R. MICHARD: I would like to draw attention to the theory of the $\mathrm{D}$ layer given by Bracewell. In this theory the $\mathrm{D}$ layer is not a Chapman region, but all the molecules responsible for the layer are ionized; then it is not necessary to postulate an enhancement of $\mathbf{L} \alpha$ emission of the order of $10^{5}$ to explain the lowering of the D layer at the time of solar flares.

C. W. Allen: The Bracewell theory is a possible explanation, but there may also be others, e.g. the existence of X-rays. 
M. Minnaert: Different upper air effects may be located at different heights, Crochets, for instance, in one layer and other effect in a different layer.

M. A. ElLISON: There is certainly evidence of two or three layers, because the relaxation times come out differently for different effects, $2.5 \mathrm{~min}$. time-lag for crochets and 7 min. for S.E.A.

T. R. KAISER: The most important far-ultra-violet atmospheric 'window' which extends into the $\mathrm{D}$ region is likely to be the narrow one which coincides with the $\mathrm{L} \alpha$ radiation. However, I would point out that there are a multiplicity of windows in the wave-length range $900-1200 \AA$ which extend to heights below $80 \mathrm{~km}$. Until more is known of the solar spectrum in this region, one should not dismiss the possibility of some contribution to $\mathrm{D}$-region effects from ultra-violet radiation other than $\mathrm{L} \alpha$.

M. Minnaert: Rocket observations during a flare would be very desirable for answering this and other questions.

\section{ASSOCIATED COSMIC-RAY EFFECTS}

\section{By H. Alfvén}

Solar flares are often associated with changes in cosmic-ray intensity, as first shown by Lange and Forbush. The change is always an increase and is distinctly different from the magnetic storm cosmic-ray effect which usually is a decrease in intensity.

The flare effect is most pronounced in the softest part of the cosmic-ray spectrum which is measured by the neutron counter technique developed by Simpson and others (the neutron counters are sensitive to primaries down to a few $\mathrm{GeV}$. whereas ionization chambers and G.M.-counters have very low sensitivity to primaries below 5 or ro GeV.). A small frare is associated with an increase of $I$ or $2 \%$, but increases by several hundred percent have been measured in connexion with big flares.

Also with ionization chambers and G.M.-counters flare effects have been observed. Only four cases are known with increases of the order Io $\%$.

Cosmic-ray effects are largest at stations situated in regions where the Earth is reached by cosmic rays coming from the direction of the Sun and moving in Störmer orbits, as shown by Firor. However, there are several cases where cosmic-ray effects have been observed far outside these regions.

The cosmic-ray effect occurs almost simultaneously with the solar flare. The time lag is often less than one hour.

It.has been claimed that an upper limit to the solar magnetic field can be obtained from the flare effect. This is true only under the assumption that the solar magnetic field is a dipole field. If, on the other hand, the solar magnetic field (or rather the interplanetary field) has the more complicated form indicated by other electromagnetic phenomena and by the fine structure of the corona, the flare effect is understandable and not in conflict with a field with a polar strength of the order ro gauss. Also, the fact that the flare effects are observed outside Firor's regions indicates the existence of an interplanetary field strong enough to scatter the particles considerably.

There are two possible interpretations of the solar flare cosmic-ray effect. Either the flare effect is the release of cosmic-ray particles trapped in magnetic fields near the Sun, or it represents an actual generation of cosmic rays. In both cases the effect is probably caused by the magneto-hydrodynamic shockwaves produced by the flare proceeding upwards through the corona.

\section{DISCUSSION}

C. B. A. McCusker: It is perhaps worth pointing out that Neher and Stern found a much weaker intensity of low energy $(<800 \mathrm{MeV}$.) particles in I954, a year of low solar activity, than in r95I. 